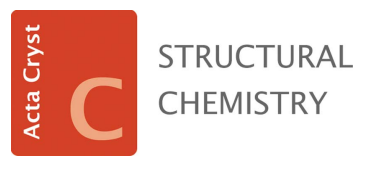

ISSN 2053-2296

\section{A new three-dimensional twofold interpenetrated cadmium(II) metal-organic framework: synthesis, structure and photoluminescence properties. Corrigendum}

\author{
Hong-Tao Zhang* and Xiao-Long Wang
}

College of Chemistry and Materials Science, Anhui Normal University, Wuhu, Anhui 241000, People's Republic of China. *Correspondence e-mail: zht2006@ahnu.edu.cn

In the article by Zhang \& Wang [Acta Cryst. (2021), C77, 691-697], the topology of the title compound is corrected.

The description of the topology of the title cadmium(II) metal-organic framework is incorrect in the article by Zhang \& Wang (2021). The description as a "new 2-nodal $(7,9)$ connected net with the point (Schläfli) symbol $\left(3^{7} \cdot 4^{6} \cdot 5^{8}\right)$ $\left(3^{8} \cdot 4^{11} \cdot 5^{16} \cdot 6\right)$ ' should be replaced by '2-nodal fsg-3,4-C2 net'. This correction should be made in the Synopsis, Abstract, at the end of the Introduction, in the final sentence of $\S 3.1$ and in the Summary. The topology determination of the title MOF was performed with ToposPro (https://topospro.com/).

Figs. 4 and 5 and their captions are updated, and the addition of the reference Blatov \& Proserpio (2009) is appropriate.

Figure 4

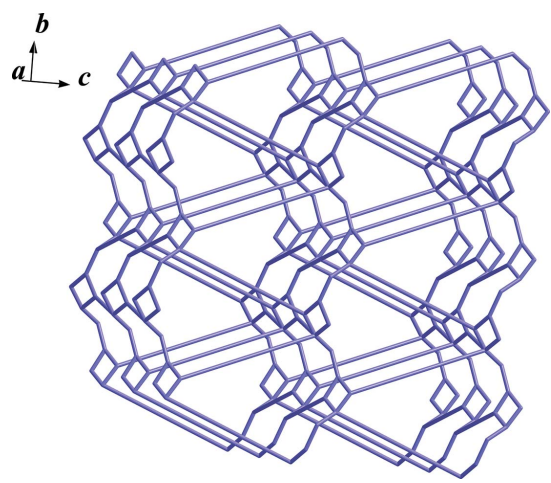

The 3,4-c net of the title MOF.

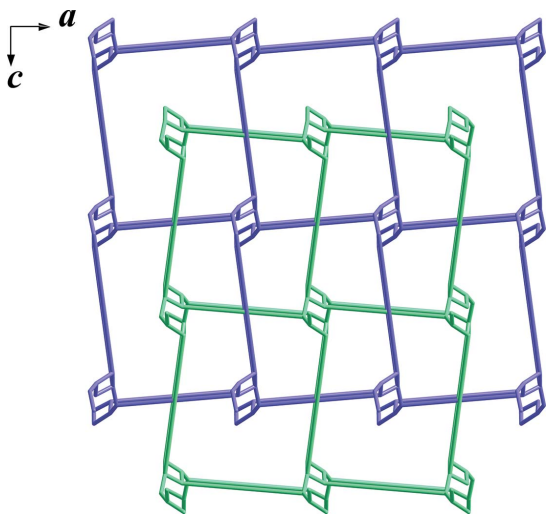

Figure 5

A simplified representation of the twofold interpenetrated 3D architecture of (I). 


\section{Acknowledgements}

We thank Professor Davide M. Proserpio for his professional advice on MOF topology.

\section{References}

Blatov, V. A. \& Proserpio, D. M. (2009). Acta Cryst. A65, 202-212.

Zhang, H.-T. \& Wang, X.-L. (2021). Acta Cryst. C77, 691-697. 